\title{
DISTRIBUIÇÃO ESPACIAL DA TUBERCULOSE E A RELAÇÃO COM AS CONDIÇÕES SOCIOECONÔMICAS NO MUNICÍPIO DE FEIRA DE SANTANA - 2005 A 2016
}

\author{
THE SPATIAL DISTRIBUTION OF TUBERCULOSIS AND RELATIONSHIP WITH THE \\ CONDIÇÕESSOCIOECONÔMICAS IN THE CITY OF FEIRA DE SANTANA - 2005-2016
}

\author{
Roquenei da Purificação Rodrigues \\ Mestre em Planejamento Territorial pela Universidade Estadual de Feira de Santana - Bahia \\ roquenei@gmail.com \\ Jocimara Souza Britto Lobão \\ Professora titular da Universidade Estadual de Feira de Santana - Bahia \\ juci.lobao@uefs.br
}

\begin{abstract}
RESUMO
Objetivou-se com esta pesquisa, realizar a distribuição espacial da incidência média dos casos novos tuberculose, em Feira de Santana - Bahia e relacionar sua ocorrência com as condições socioeconômicas. Para tanto, foram incluídos no estudo, os casos novos de tuberculose notificados no município de Feira de Santana - Bahia no período de 2005 -2016. Inicialmente foi calculada a incidência média dos casos e para correlacionar esta morbidade e as variáveis socioeconômicas dos bairros e distritos rurais, usou-se o modelo de regressão de Poisson. Tais variáveis foram obtidas a partir do Censo demográfico do IBGE, 2010. Os resultados apontaram que se teve uma maior concentração da incidência média nos bairros centrais. Com relação à associação da incidência média da tuberculose com as variáveis socioeconômicas, verificou- se associações diretas entre incidência da doença e a proporção de domicílios com 11 ou mais pessoas, proporção de domicílios com lixo coletado por serviço de limpeza, com rede geral de abastecimento e sem banheiro ou sanitário. Concluiu-se que a distribuição espacial da tuberculose ocorre de forma heterogênea e que nos bairros com baixa vulnerabilidade social, apresentam uma alta taxa de incidência média da patologia. O que se leva a necessidade de novos estudos para elucidar essa questão.
\end{abstract}

Palavras- Chave: Tuberculose. Distribuição espacial. Vulnerabilidade socioeconômica.

\begin{abstract}
It was objectified with this research, to carry through the space distribution of the average incidence of the new cases tuberculosis, in Fair of Santana - Bahia and to relate its occurrence with the socioeconômicas conditions. For in such a way, they had been enclosed in the study, the new cases of tuberculosis notified in the city of Fair of Santana - Bahia in the period of $2005-2016$. Initially the average incidence of the cases was calculated and to correlate this morbidade and the socioeconômicas 0 variable of the quarters and agricultural districts, the model of regression of Poisson was used. Such 0 variable had been gotten from the demographic Census of IBGE, 2010. The results had pointed that a bigger concentration of the average incidence in the quarters was had central offices. With regard to the association of the average incidence of the tuberculosis with the socioeconômicas 0 variable, it verified direct associations between incidence of the illness and the ratio of domiciles with 11 or more people, ratio of domiciles with garbage collected for cleanness service, with general net of supplying and without bathroom or bathroom. It was concluded that the space distribution of the tuberculosis occurs of heterogeneous form and that in the quarters with low social vulnerability, present one high tax of average incidence of the pathology. What if it takes the necessity of new studies to elucidate this question.
\end{abstract}

Key words: Tuberculosis. Spatial distribution. Socioeconomic vulnerability.

Recebido em: 06/01/2020

Aceito para publicação em: 17/03/2020 


\section{INTRODUÇÃO}

A tuberculose (TB) é uma afecção infectocontagiosa, considerada uma das mais antigas da história da humanidade e é desencadeada pelo Mycobacterium tuberculosis que acomete diversos órgãos do corpo, principalmente os pulmões. Tal patologia representa um problema de saúde pública mundialmente e de acordo com a Organização Mundial de Saúde (OMS), no ano de 2018, calcula-se que ocorreram 10,0 milhões de casos novos de tuberculose (WHO, 2019). Neste mesmo ano, foram notificados 72.788 casos novos de tuberculose no Brasil, sendo na Bahia, 4.750 casos e em Feira de Santana, 229 ocorrências (SVS, 2019; SMS, 2019).

Dada a sua importância epidemiológica, é importante considerar que além da questão biológica, a TB possui uma dinâmica associada às condições precárias de vida como: moradia inadequada, desnutrição, dificuldade de acesso aos serviços e bem públicos, sobretudo o de saúde (BRASIL, 2014). Além disso, há de se considerar que ainda existe na sociedade, tabus e crenças, que dificultam ações preventivas e curativistas (SANTOS et al.,2018).

Nesta perspectiva, vale destacar que para se traçar o perfil e ações de controle da TB e de outras doenças, é necessário compreender como os eventos patológicos se distribuem no espaço, associado com os fatores determinantes e condicionantes. Desta forma, o geoprocessamento, por meio dos Sistemas de Informação Geográficas (SIG) e a análise espacial, são elementos que contribuem para a identificação das áreas geográficas e grupos da população que apresentam maior risco de adoecer ou morrer, além de favorecer a reorganização de serviços de saúde e intervenções sociais (BRASIL, 2006; OLIVEIRA;FONZAR; SENTIL, 2017) . Dentre as técnicas que permitem essa compreensão, destacam-se as seguintes: mapeamento de doenças, técnicas de aglomeração de casos, estudos da difusão, identificação de fatores de risco por comparação de mapas e análise de regressão (SANTOS et al, 2014).

O presente estudo teve como objetivo realizar a distribuição espacial da incidência média dos casos novos tuberculose, em Feira de Santana - Bahia e relacionar sua ocorrência com as condições socioeconômicas.

Vale ressaltar, que casos novos de tuberculose corresponde a notificação de pessoas que nunca foram submetidas à quimioterapia antituberculosa, fez uso de tuberculostáticos por menos de 30 dias, ou que realizaram o tratamento da TB há cinco anos ou mais (BRASIL, 2011).

\section{METODOLOGIA}

\section{- Tipo de estudo}

Trata-se de um estudo epidemiológico, ecológico, de base territorial. Conforme Rouquayrol e Almeida Filho (2003), os estudos ecológicos abordam áreas geográficas bem delimitadas, analisando comparativamente variáveis globais, quase sempre por meio da correlação entre indicadores de condições de vida e indicadores de situação de saúde.

\section{- Campo de Estudo}

O município de Feira de Santana é considerado um potente centro econômico do interior do Estado da Bahia, possui uma extensão territorial que abrange $1.304,425 \mathrm{Km}^{2}$, dividido em 44 bairros (área urbana) e sete distritos rurais (Figura 1). Conforme o Censo demográfico de 2010, realizado pelo Instituto Brasileiro de Geografia e Estatística (IBGE), a população era de 556.642 pessoas. Sendo que, foi estimado para o ano de 2019, um número de 614.872 habitantes (IBGE, 2020).

\section{- Fonte de Dados}

Foram selecionados os dados referentes aos casos novos de tuberculose, na forma pulmonar e extra-pulmonar, entre os anos de 2005 - 2016, dos indivíduos residentes em Feira de Santana e que constava na notificação, o bairro ou distrito rural de origem. Os dados foram extraídos do Sistema Nacional de Agravos de Notificação - SINAN e disponibilizados pela Vigilância Epidemiológica municipal. 
Os dados sobre o número da população residente nos bairros e distritos rurais e as características sociodemográficas: percentual de pessoas com até um salário mínimo; de domicílios com 11 ou mais pessoas; de domicílios com lixo coletado por serviço de limpeza; com rede geral de abastecimento de água e sem banheiro ou sanitário) foram obtidas na base de dados do IBGE, conforme o Censo de 2010.

Figura 1 - Município de Feira de Santana- Bahia, com a divisão da área urbana (bairros) e distritos rurais, conforme o Censo do IBGE, 2010

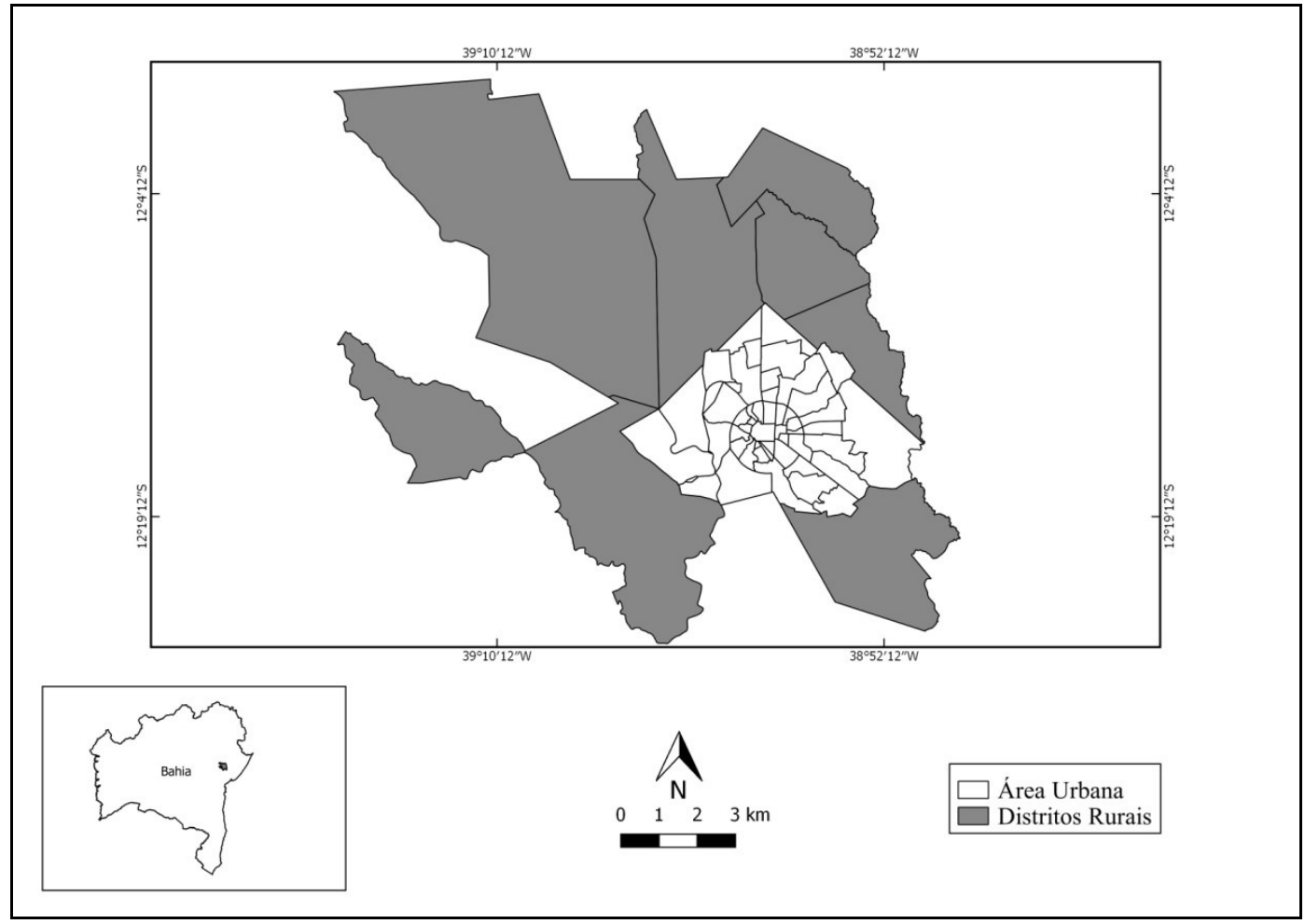

Fonte: IBGE, 2010

Elaborado por Roquenei da Purificação Rodrigues, 2018

\section{- Análise dos dados}

Os dados referentes ao número de casos notificados por bairro e distrito rural e da população destes foram tabulados no Microsoft ${ }^{\circledR}$ Office Excel, versão 2016 e em seguida foi calculada a taxa de incidência média.

Tal taxa foi calculada com base no estudo de Santos (2016), em que o numerador corresponde ao total de casos da tuberculose, dividido por 11(total de anos do estudo) e no denominador a população de cada bairro ou distrito rural conforme o último censo demográfico. Logo:

Incidência média $=\left(\mathrm{N}^{\circ}\right.$ casos novos de $\left.\mathrm{TB} / 11\right) \times 100.000$ hab. População do bairro ou distrito rural pelo (Censo 2010) 
As análises estatísticas foram realizadas no software STATA (versão 13.0, STATA Corp., College Station, Texas). Os dados foram analisados por modelo linear generalizado (GLM) com distribuição de Poisson e função de ligação log. A variável dependente foi taxa de incidência da tuberculose pulmonar por 100.00 habitantes segundo os 44 bairros e os oito distritos rurais do município, entre os anos já mencionados. As variáveis explicativas foram os dados socioeconômicas do Censo 2010: percentual de pessoas com até um salário mínimo; percentual de domicílios com 11 ou mais pessoas; percentual de domicílios com lixo coletado por serviço de limpeza; percentual de domicílios com rede geral de abastecimento de água e sem banheiro ou sanitário. Estes dados foram armazenados em planilha eletrônica e foi verificada a consistência dos dados para as análises.

As variáveis explicativas foram testadas separadamente. Aquelas que apresentaram valor $p$ menores que 0,20 ) foram incluídas no modelo múltiplo. Para tanto, foi utilizado o método stepwise forward selection na análise de regressão, em que, por meio de um modelo mais simples - aquele só com uma variável socioeconômica -, chegou-se a um modelo mais complexo, no qual foram inclusas todas as variáveis socioeconômicas significantes. Em todas as análises, utilizou-se o nível de significância de $5 \%$.

O teste de multicolinearidade Variance Inflation Factor entre as variáveis independentes, com tolerância inferior a 10 foi realizado previamente à análise múltipla. A seleção do modelo múltiplo com melhor ajuste foi realizada pelo teste da razão de verossimilhanças, que consiste em um teste de hipótese que compara a qualidade do ajuste entre dois modelos, em que um dos modelos é subconjunto do outro.

\section{- Distribuição espacial dos dados}

Após o cálculo da incidência média dos casos novos de tuberculose, os dados foram armazenados em ambiente de Sistema de Informação Geográfica, por meio do software Qgis, versão 2.18 e os mapas foram elaborados usando o modo de classificação Quantil.

\section{- Questões éticas}

Os dados foram obtidos de fonte secundária, sem a identificação nominal dos sujeitos, e usados de forma agregada, de modo que foi descartado o parecer por um Comitê de Ética em Pesquisa, conforme o art. $1^{\circ}$ da Resolução Conselho Nacional de Saúde nº 510/2016.

\section{RESULTADOS}

Foram notificados no período de 2005-2016, no município de Feira de Santana - Bahia, 2.266 casos novos de tuberculose. Destes casos, 1.731 constavam em registro, o bairro ou o distrito rural em que o infectado residia.

A figura 2 mostra a distribuição espacial da incidência média dos casos de tuberculose. As variáveis visuais,em escala cinza, variando a intensidade de acordo a sequência de valores apresentados nas classes estabelecidas. No período estudado, os bairros com as maiores incidências foram: Aviário, Subaé, Santo Antônio dos Prazeres, Novo Horizonte, Pampalona, Capuchinhos, Parque Getúlio Vargas, Ponto Central, Centro, Rua Nova e Sobradinho. Com relação aos distritos rurais, o que teve maior incidência foi o distrito Governador Dr. João Durval Carneiro. 
Figura 2 - Distribuição espacial, da incidência média por 100.000 habitantes, nos bairros e distritos rurais de Feira de Santana- Bahia, entre os anos de 2005- 2016

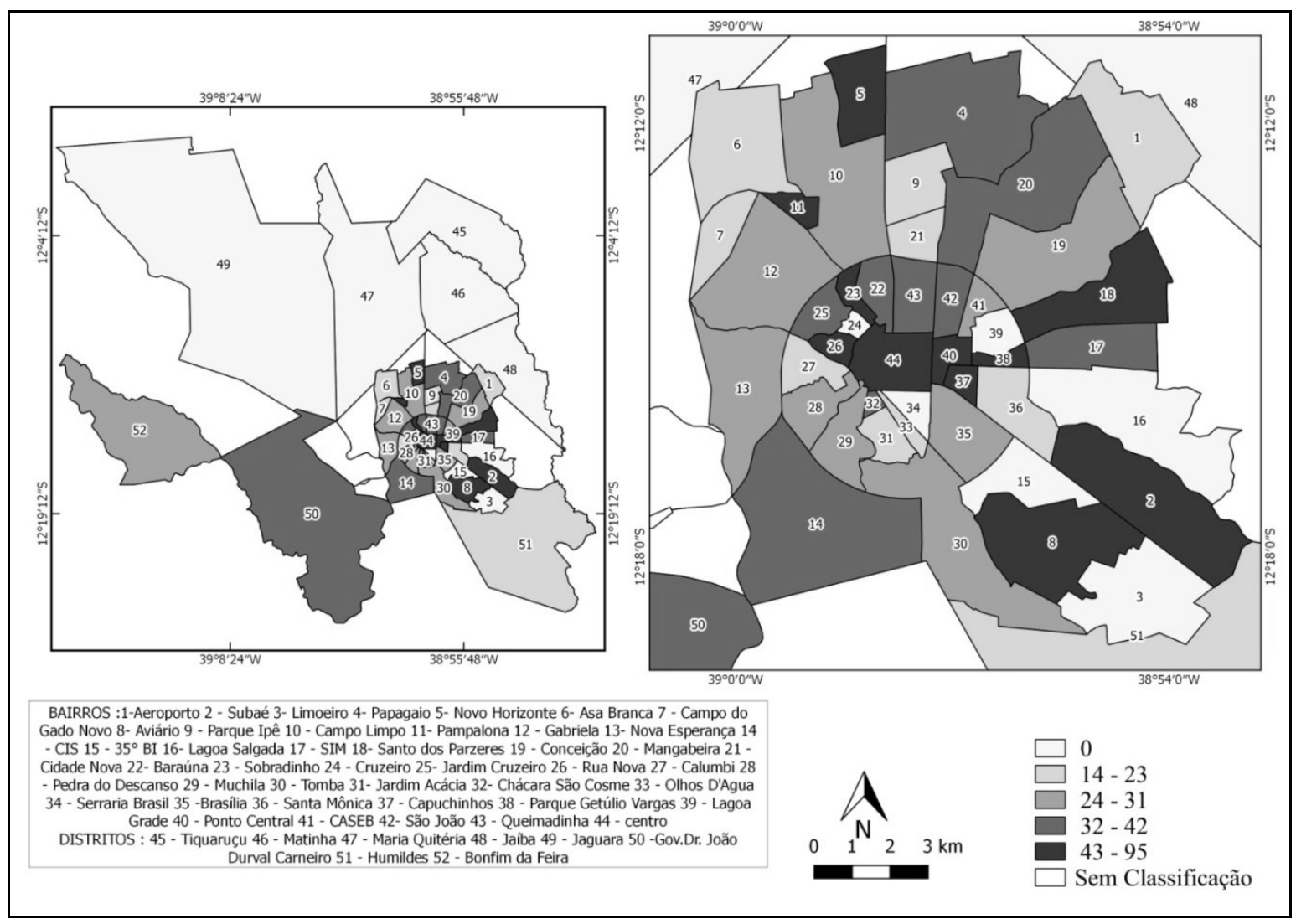

Fonte: SMS, 2017; IBGE, 2010

Elaborado por Roquenei da Purificação Rodrigues, 2018

A figura 3 apresenta o percentual das variáveis socioeconômicas conforme o Censo do IBGE, 2010.

No mapa A, observa-se a existência de um morfema axial para a variável renda até 1 salário mínimo. Esse morfema indica que, partindo do centro da cidade de Feira de Santana, a renda da população tende a cair, radialmente, em direção à periferia e distritos rurais. Em relação ao mapa B, nota-se um morfema axial parecido. A maior parte das regiões com mais de 11 moradores por residência, encontra-se na periferia da zona urbana.

No mapa C, há uma concentração de informações analisadas, percentual de domicílios sem banheiro e ou sanitário, na região Sudoeste e Sudeste da cidade. O mapa D, por sua vez, apresenta uma situação conflitante, do ponto de vista infraestrutural, com as informações do mapa $\mathbf{C}$. Pois, observa-se no mapa $\mathbf{D}$, que existe uma concentração de residências com acesso à água tratada na região Sudoeste. Todavia, nesta região também há um problema com oferta de residências sem banheiro e ou sanitário. No mapa $\mathbf{E}$, verifica-se que o maior percentual de limpeza por serviço público, dá-se principalmente nos bairros centrais. 
Figura 3 - Distribuição espacial, das variáveis socioeconômicas conforme o Censo do IBGE
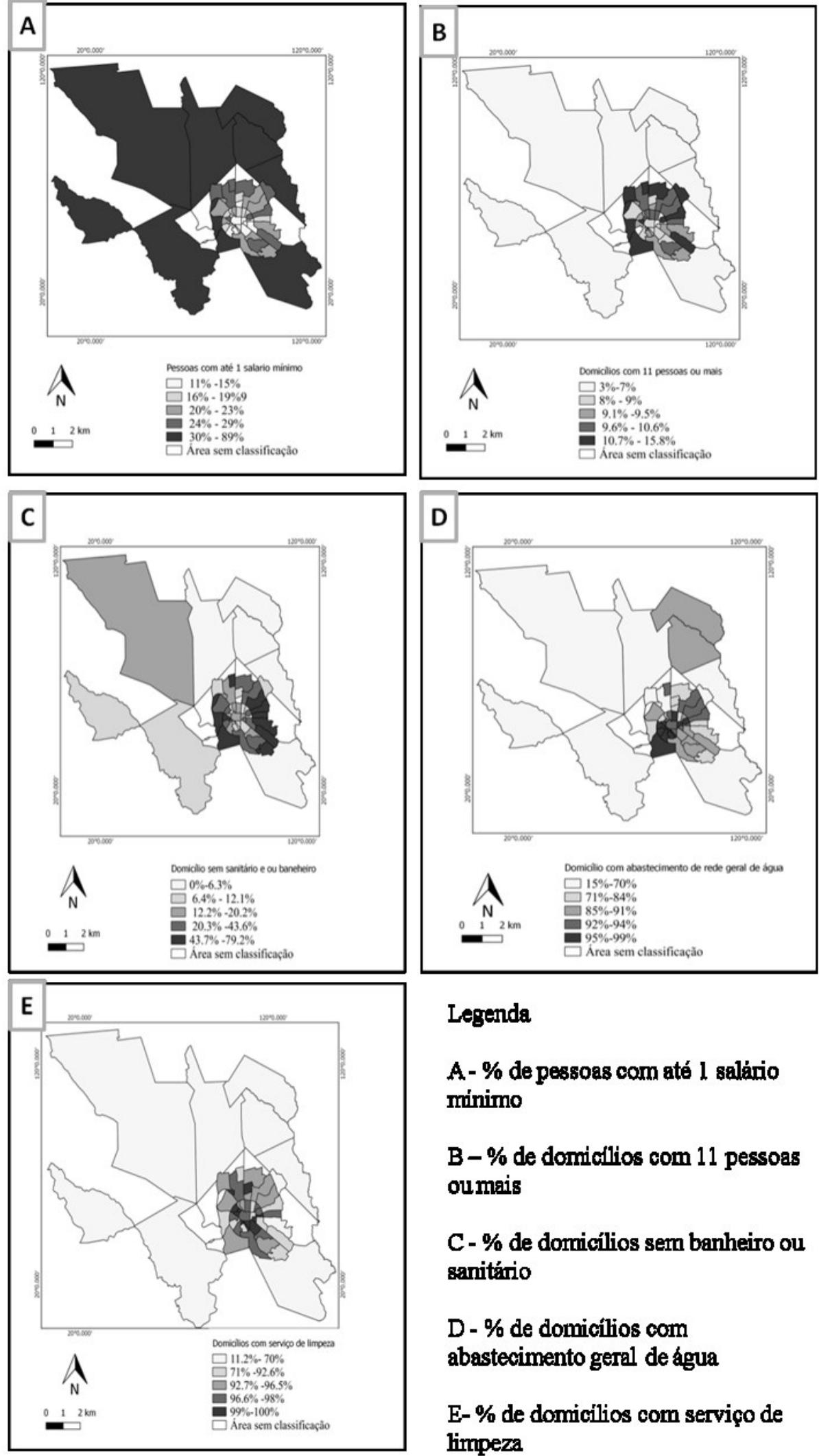

\section{Legenda}

A - \% de pessoas com até 1 salário mínimo

\section{B-\% de domiclitios com 11 pessoas oumais}

C- \% de domicílios sem banheiro ou sanitário

\section{D - \% de domicilios com} abastecimento geral de água

E-\% de domicilios com serviço de limpeza

Fonte: IBGE, 2010

Elaborado por Roquenei da Purificação Rodrigues, 2018 
$\mathrm{Na}$ tabela 1, são apresentados os resultados da regressão de Poisson, tendo como desfecho a variável incidência média da tuberculose e como variáveis explicativas os dados relativos a condições socioeconômicas do Censo 2010 do IBGE.

Tabela 1 - Parâmetros da análise bivariada e múltipla com as respectivas razões de taxa de incidência de tuberculose segundo bairros e distritos rurais

\begin{tabular}{lcccc}
\hline & \multicolumn{2}{c}{ Bivariada } & \multicolumn{2}{c}{ Múltipla } \\
Variáveis socioeconômicas & IRR & IC95\% & IRR & IC95\% \\
\hline \% pessoas com até 1 SM & $0,29^{*}$ & $0,22-0,39$ & $0,61^{*}$ & $0,37-0,99$ \\
\% de domicílios com 11 ou mais pessoas & $217,78^{*}$ & $54,51-$ & $11,13^{*}$ & $1,60-$ \\
& & 870,04 & & 77,67 \\
\% domicílios com lixo coletado por serviço de & $3,25^{*}$ & $2,47-4,26$ & $1,81^{*}$ & $1,19-2,74$ \\
limpeza & & & & \\
\% domicílios com rede geral de abastecimento & $2,51^{*}$ & $1,83-3,45$ & - & \\
\% domicílios sem banheiro ou sanitário & $1,38^{*}$ & $1,09-1,75$ & - & \\
\hline
\end{tabular}

IRR: Incidence Rate Ratio (Razão de Taxas de Incidência) ${ }^{*} \mathrm{p}<0,05$

Fonte: IBGE, 2010

Elaborado por Roquenei da Purificação Rodrigues, 2018

\section{DISCUSSÃO}

Este é o primeiro estudo realizado no município de Feira de Santana- Bahia, objetivando descrever a distribuição espacial das taxas de incidência média dos casos novos de tuberculose. Tal município é considerado um dos 23 , do estado da Bahia, primados para o Programa Nacional de Controle da Tuberculose, visando à inserção das ações de educação em saúde, comunicação e mobilização social, conforme o contexto de cada localidade, buscando uma melhor adesão ao tratamento, reduzir o preconceito que ainda existe a respeito desta morbidade e fortalecer a vigilância dos contatos, para que não se tenha evasão da terapêutica (BRASIL, 2004).

Conforme Hino; Santos; Villa (2005), a qualidade da informação da variável endereço e a eficiência do Sistema de Informação Geográfica para localizar os eventos pontualmente é essencial para possibilitar as análises dos padrões de distribuição dessas ocorrências. Embora neste estudo, não se tenha trabalhado com o endereço de forma pontual, e sim por polígonos, verificou-se que a variável em questão, correspondente ao bairro ou distrito rural, em que os infectados residiam, ou não foram preenchidas em todas as fichas de notificação, por parte do profissional de saúde, ou teve falha da equipe técnica em realizar o lançamento dos dados, no Sistema de Informação de Agravos de Notificação.

Romero et al. (2016) apontam que as deficiências no preenchimento da ficha de notificação, inobservância dos prazos de envio e fragilidade no fluxo de informação entre as unidades notificadoras e a vigilância epidemiológica, podem comprometer um monitoramento seguro da realidade apresentada.

Nesta perspectiva, Vendramini et al. (2005), destacam em seu estudo, que a identificação de áreas com diferentes riscos para TB, permite que o sistema de saúde municipal trate o município como composto de distintas realidades e priorize as regiões com as maiores incidências da doença. Vale destacar observando a figura 2, que o bairro Aviário está na classe de maior incidência e é lá que se localiza o presídio. Estudos anteriores (Caborne et al,2017; Reis et al,2016; Estevan, Oliveira e Croda, 2013) revelam que a população carcerária é mais vulnerável a enfermidades desta natureza. Por outro lado nos bairro Subaé e Centro Industrial do Subaé, localizam-se duas áreas com grande concentração de indústrias. Ou seja, o $\mathrm{CO}^{2}$ lançado pelas chaminés contribui para o agravo de doenças respiratórias e associado às precárias condições higiênicas sanitárias e econômicas tornam os indivíduos mais suscetíveis a contrair a TB.

Os bairros localizados no centro da cidade, embora com os maiores índices de condições de vida, possuem alta taxa da incidência média dos casos novos de TB. Como por exemplo, nos bairros Santa Mônica, Capuchinhos, Ponto Central e Centro.Fato que pode ser explicado com o adensamento de uma população flutuante, pois neles se concentram o comércio da cidade além de micro zonas com indivíduos em situação antípoda de vida. Os demais bairros com maior incidência 
coincidem com grande número de população de baixa renda a exemplo do Novo Horizonte e Pampalona.

Com relação aos indicadores socioeconômicos e a TB, os domicílios com rede geral de água, encontrou-se uma associação inversa entre a oferta do serviço e a redução da incidência média da TB. Esta mesma associação ocorreu também com os indicadores: coleta de lixo por serviço público e residência com banheiros e sanitários.

Estes resultados representam uma controvérsia nos achados da literatura, como pode ser verificado no estudo de Vendramini et al.(2010), Hino et al. (2011) e San Pedro e Oliveira, (2013), uma vez que, as precárias condições destes indicadores, representam uma estreita relação com as altas taxas de incidência da tuberculose. No entanto, vale considerar que embora o município de Feira de Santana, apresente uma social e espacial, existe um alto percentual de cobertura, nos bairros, para as variáveis: abastecimento de água por rede geral e coleta de lixo por serviço. O que pode justificar a relação negativa entre oferta destes serviços e a redução das taxas da incidência média da referida doença. Além disso, o ambiente residencial pode ser mais importante do que o ambiente do bairro para a incidência da tuberculose.

Ao realizar um estudo sobre o impacto das condições de vida e educacional, em todas as regiões do Brasil, Teixeira e Costa (2011) referem que se uma habitação não possui banheiro ou sanitário, menor é a higiene e maiores são as chances de se contrair tuberculose. Neste estudo, as áreas com maior número de banheiro e ou sanitário, não representaram atenuação da incidência média da TB.

Com relação à renda, ter grande influência na ocorrência da TB, verificou-se por meio do modelo de regressão adotado, não foram encontrados valores significativos entre os indivíduos que recebiam até 1 salário mínimo, e o aumento da incidência da patologia em questão, nos bairros e distritos rurais. Todavia, a literatura corrobora que os indivíduos com baixa renda, têm uma condição avessa, pois os expõem às questões desiguais de ter acesso a serviços médicos e ter uma boa alimentação, tornando-os mais vulneráveis para o adoecimento e para o abandono do tratamento da TB (VENÂNCIO; TUAN; NASCIMENTO, 2014).

Fazendo referência ao número de pessoas por domicílio, verificou-se neste trabalho, uma estreita relação entre os domicílios com 11 pessoas ou mais, e a ocorrência da TB. Siqueira (2014), frisa que plausibilidade biológica da relação pode ser explicada pelo fato do maior adensamento de pessoas, em especial nos locais de residência, aumentar a exposição a patógenos cujomodo de transmissão se faz por via aérea, a exemplo do Mycobacterium tuberculosis.

Embora não fosse investigado neste estudo, a relação de domicílios adequados e inadequados, os achados na literatura enfocam que muitos domicílios são pouco ventilados, pequenos, escuros e desta forma predispõem a transmissibilidade do bacilo da TB (VICENTIN, SANTO; CARVALHO, 2002).

\section{CONSIDERAÇÕES FINAIS}

As limitações do estudo estiveram relacionadas ao uso de dados secundários que, muitas vezes, apresentam-se inconclusos, desatualizados e com inconsistências. Todavia, os achados permitiu identificar que a incidência média dos casos novos de TB, distribui-se de forma heterogênea e diferente dos achados da literatura, as maiores ocorrências se deus nos bairros centrais onde se têm as melhores condições socioeconômicas.

Sugere-se que novos estudos sejam realizados sobre a temática proposta, para melhor elucidar as desigualdades sociais, econômicas, sanitárias e de saúde no município de Feira de Santana -Bahia, que contribuem para a ocorrência da Tuberculose. Desta forma, pode-se traçar ações de saúde de forma intersetorial, como forma de melhoria da qualidade de vida da população e minimização dos casos desta patologia. 


\section{REFERÊNCIAS}

BRASIL. Ministério da Saúde Secretaria de Vigilância em Saúde. Departamento de Vigilância Epidemiológica. Brasília: Ministério da Saúde, 2004.

BRASIL. Ministério da Saúde. Secretaria de Vigilância em Saúde. Fundação Oswaldo Cruz. Abordagens espaciais na saúde pública. Brasília: Ministério da Saúde, 2006.

BRASIL. Ministério da Saúde. Secretaria de Vigilância em Saúde. Departamento de Vigilância Epidemiológica. Manual de recomendações para o controle da tuberculose no Brasil / Ministério da Saúde, Secretaria de Vigilância em Saúde, Departamento de Vigilância Epidemiológica. - Brasília: Ministério da Saúde, 2011.

CARBONE, A.S.S.; et al. Estudo multicêntrico da prevalência de tuberculose e HIV na população carcerária do estado do Mato Grosso do Sul. Comunicação em Ciências da Saúde, v. 28 , n. 01, p.53-57, 2018.

ESTEVAN, AO; OLIVEIRA, SM; CRODA, J. Active and latent tuberculosis in prisoners in the Central-West Region of Brazil. Revista da Sociedade Brasileira de Medicina Tropical. 2013;46(4):515-8. https://doi.org/10.1590/0037-8682-1441-2013

HINO, P; SANTOS C.B., VILLA, T.C.S. Evolução espaço-temporal dos casos de tuberculose em Ribeirão Preto (SP), nos anos de 1998 a 2002. J Bras Pneumol 2005; 31(6):523-527. https://doi.org/10.1590/S1806-37132005000600011

HINO, P.; et al. Controle da tuberculose na perspectiva da vigilância da saúde. Esc Anna Nery (impr.). 2011; 15(2): 417-42. https://doi.org/10.1590/S1414-81452011000200027

IBGE - Instituto Brasileiro de Geografia e Estatística. Extraído de http:// www. ibge.gov.br/cidadesat/default.php. Acesso em agosto de 2020.

OLIVEIRA, A.; FONZAR, U.J.V; SANTIL, F.L.P. Análise espacial da tuberculose nos anos de 2010 a 2015 - na cidade de Maringá-PR. Geoingá: Revista do Programa de Pós-Graduação em Geografia Maringá. , 2017; v. 9, n. 2, p. 52-70.

REIS, A.J; et al. Transmissão recente de Mycobacterium tuberculosis resistentes aos antimicrobianos em população carcerária no sul do Brasil. J Bras Pneumol. 2016; 2229(6): 633-9.

ROMERO, R.O.G et al.,. Subnotificação de casos de tuberculose a partir da vigilância do óbito. Rev Eletr Enferm. 2016 jun;18:e1161. https://doi.org/10.5216/ree.v18.37249

ROUQUAYROL, M.Z; ALMEIDA - FILHO, N. Epidemiologia \& Saúde. $6^{\text {a }}$ edição. Rio de Janeiro: MEDSI,2003

SAN PEDRO, A.; OLIVEIRA, R.M. Tuberculose e indicadores socioeconômicos: revisão sistemática da literatura. Rev. Panam Salud Publica, 2013; 33(. 4): 294 -301. https://doi.org/10.1590/S1020-49892013000400009

SANTOS, N.S.G.M, et al. Tuberculose e análise espacial: Revisão da literatura. Ciência y enfermeira. 2014; 20(2): 117-29. https://doi.org/10.4067/S0717-95532014000200012

SANTOS,W. et al. , Representações Sociais de pessoas com tuberculose pulmonar sobre enfrentamentos diante dos conflitos sociais e pessoais. Rev. Bras. Pesq. Saúde, Vitória, 20(1): 6-13, jan-mar, 2018. https://doi.org/10.21722/rbps.v20i1.20603

SMS- Secretaria Municipal de Saúde de Feira de Santana. Boletim Epidemiológico da Tuberculose. 2019; edição 01,p.1-2.

SVS - Secretaria de Vigilância em Saúde. Brasil Livre da Tuberculose: evolução dos cenários epidemiológicos e operacionais da doença. 2019; vol.50, n 9, p.1-18.

SILVA, A.P.S.C. Duas décadas de tuberculose em uma cidade do Nordeste Brasileiro: avanços e desafios no tempo e no espaço.Tese (doutorado em saúde pública) - Centro de Pesquisas Aggeu Magalhães, Fundação Oswaldo Cruz, Recife. 2016.

SIQUEIRA, A.S.P. Determinantes socioeconômicos da produção da tuberculose: um estudo no município de Itaboraí, Região Metropolitana do Rio de Janeiro, no período de 2000 a 
2011. Tese (Doutorado) - Escola Nacional de Saúde Pública Sergio Arouca, Rio de Janeiro, 2014.

WORLD HEALTH ORGANIZATION. Global tuberculosis report 2019 [Internet]. Geneva: World Health Organization; 2019. . Available from: http:/l https://apps.who.int/iris/bitstream/handle/10665/329368/9789241565714-eng.pdf. Acesso em: Março de 2020.

TEIXEIRA, E.C.C, COSTA, J.S. O impacto das condições de vida e da educação sobre a incidência de tuberculose no Brasil. Rev Econ. 2011, 37 (2): 106-123. https://doi.org/10.5380/re.v37i2.27241

VENANCIO, T.S, TUAN, T.S, NASCIMENTO, L.F.C. Indidence of tuberculosis in children in the state of São Paulo, Brazil, under spatial approach. Ciênc Saúde Coletiva. 2015; 20(5):1541-7. https://doi.org/10.1590/1413-81232015205.14672014

VENDRAMINI S.H.F, et al. Tuberculose em município de porte médio do sudeste do Brasil: indicadores de morbidade e mortalidade, de 1985 a 2003. J Bras Pneumol. 2005; 31(3):23743. https://doi.org/10.1590/S1806-37132005000300010

VENDRAMINI, S. H. F. et al.; Análise espacial da co-infecção tuberculose/HIV: relação com níveis socioeconômicos em município do sudeste do Brasil. Rev Soc Bras Med Trop, Ribeirão Preto, SP,2010, v. 43, n. 5, p. 536-541. https://doi.org/10.1590/S0037$\underline{86822010000500013}$

VICENTIN, G.; SANTO, A. H.; CARVALHO, M. S.; Tuberculosis mortality and social indicatory in Rio de Janeiro city. Ciênc. Saúde Coletiva, vol.7, n.2, p.253-263, 2002. https://doi.org/10.1590/S1413-81232002000200006 